\title{
SDHB mutated paraganglioma imitating thyroid tumor: A case report and review of literature
}

\author{
Vygante Maskoliunaite*1, Gabija Makunaite ${ }^{2}$, Ausra Garnelyte ${ }^{1,2}$, Agne Abraitiene ${ }^{3}$, Virgilijus Beisa ${ }^{4}$ \\ ${ }^{1}$ National Center of Pathology, Affiliate of Vilnius University Hospital Santaros Klinikos, Lithuania \\ ${ }^{2}$ Faculty of Medicine, Vilnius University, Lithuania \\ ${ }^{3}$ Centre of Endocrinology, Vilnius University Hospital Santaros Klinikos, Lithuania \\ ${ }^{4}$ Centre of Abdominal Surgery, Vilnius University Hospital Santaros Klinikos, Lithuania
}

Received: May 27, 2018

DOI: $10.5430 /$ crcp.v5n2p5
Accepted: July 24, 2018

Online Published: August 28, 2018

\begin{abstract}
Paragangliomas of the head and neck are uncommon tumors arising from parasympathetic ganglia. Paragangliomas are mostly asymptomatic and may manifest as palpable mass of neck. The morphologic features are non-specific and comparable to the other neuroendocrine tumors. Most of hereditary cases are associated with alterations in genes of succinate dehydrogenase (SDH). SDHA and SDHB immunohistochemistry is considered as reliable screening method to detect tumors with genetical alterations. Of note, SDHB mutated paragangliomas have the highest risk of local recurrence, distant metastasis and the development of other tumor phenotypes, which are associated with mutation. Therefore, active surveillance of patients and early surgical treatment are essential. In contrast, SDHB mutated head and neck paragangliomas was considered as completely benign tumors, although, the latest literature describes more controversial cases, which may increase awareness. Here, we present a rare case of 21 years old female with asymptomatic neck paraganglioma, which was unexpectedly diagnosed after pathological and immunohistochemical testing of removed thyroid gland and showed unusual immunohistochemical variation for SDH mutation.
\end{abstract}

Key Words: Paraganglioma, Neuroendocrine tumor, Neck, SDH, Immunohistochemistry

\section{INTRODUCTION}

Head and neck paragangliomas (PGs) originate from the ganglia of the parasympathetic system. $60 \%$ of these cases arise in carotid body, the remaining cases are localized in middle ear $(30 \%)$, along the vagal nerve (10\%) or occasionally in larynx. ${ }^{[1]}$ Most of tumors are asymptomatic and may be detected as palpable, painless mass of neck. ${ }^{[1,2]}$ Less than $1 \%$ of head and neck PGs are functional with catecholamine production. A slight female predominance is noticed. Mostly, these tumors arise in 5th-6th decade, but earlier presence is more frequently associated with hereditary syndromes. ${ }^{[1-3]}$
The preoperative diagnosis of neck PGs may be difficult because of its non-specific radiological appearance and low hormonal activity. Fine needle aspiration is mostly nondiagnostic. ${ }^{[2-4]}$ The precise frozen section diagnosis may be complicated because the morphology of the tumor is often disorganized and histological features like sustentacular cells are often unrecognizable. Therefore, these tumors tend to be misdiagnosed intraoperatively. The diagnosis of PGs is usually confirmed after surgery by complete histological and immunohistochemical evaluation. ${ }^{[5]}$

Histologically, the majority of PGs are composed of rounded

\footnotetext{
*Correspondence: Vygante Maskoliunaite; Email: vygantem@gmail.com; Address: National Center of Pathology, Affiliate of Vilnius University Hospital Santaros Klinikos, Lithuania.
} 
cells with clear cytoplasm and almost always with welldeveloped nested architecture of "zellballen" pattern which is surrounded by a prominent vasculature and thin fibrotic capsule. Non-specific morphologic appearance may cause difficulties to diagnose PGs without immunohistochemical testing. The tumor cells are positive for neuroendocrinal markers, negative for keratins and the sustentacular cells are positive for S100. ${ }^{[3,6]}$ Paragangliomas can be misdiagnosed with the others neuroendocrine-morphology resembling tumors like medullary thyroid carcinoma. ${ }^{[2,5]}$

When diagnosis of paraganglioma is established, a testing for hereditary syndromes must be considered, because paragangliomas and pheochromocytomas (PG/PHEO) are among the most highly hereditary of all neoplasm. At least one third of these tumors are associated with proved germline mutations. ${ }^{[1,3,6,7]}$ Approximately $15 \%$ of all PG/PHEO have autosomal dominant germline mutations in succinate dehydrogenase (SDH) subunit genes such as SDHA, SDHB, SDHC, SDHD and SDHAF2 (a flavination and assembly factor). ${ }^{[8]}$ These patients have higher risk for multiple PG/PHEO, also, renal cell carcinomas (RCC), gastrointestinal stromal tumors (GIST) and pituitary adenomas. ${ }^{[3,6]}$ Of note, SDHB mutated paragangliomas have been associated with higher rate of metastasis (from $30 \%$ to $71 \%$ ). In contrast, only $3 \%$ of SDHB non-mutated tumors are metastatic. ${ }^{[8,9]}$ SDHA and SDHB immunohistochemistry is considered as reliable method to select patients for genetic consultation. Of note, the clinical course of SDHB mutated head and neck paragangliomas still remains inconsistent. Formerly, it was shown, that patients harboring SDHB mutations had unilateral lateonset head and neck paragangliomas without evidence of recurrence or malignancy. ${ }^{[10]}$ In the latest literature SDHB negative head and neck paragangliomas not only metastasized, occurred for younger patients but also had variable manifestation of the SDHB-linked tumor syndrome, including catecholamine producing pheochromocytomas. ${ }^{[1,12]}$ Of these reasons, SDHB mutated head and neck paragangliomas may require a long-term follow-up for patients and testing for their family members as well as SDHB mutated tumors in other localizations. ${ }^{[1,3,12-14]}$

\section{Case presentation}

A 21-year-old female with palpable neck nodule, which was noticed 2 years ago, was subjected to ultrasound examination. A hypoechogenic, well-vascularized, $19 \mathrm{~mm} \times 10 \mathrm{~mm}$ in diameter structure, comparable to thyroid tissue was detected above the isthmus of thyroid. The American College of Radiology (ACR) TI-RADS system was used to evaluate likelihood of malignancy according to ultrasound findings of thyroid nodules. It revealed mildly suspicious nodule because of hypoechogenicity (see Figure 1). Technetium $99 \mathrm{~m}$ pertechnetate thyroid scan and a single-photon emission computerized tomography (SPECT) showed normal thyroid tracer uptake $(0.7 \%)$. SPECT images showed tracer uptake above the right thyroid lobe and isthmus, considered as uptake in the pyramidal lobe (see Figure 2). The diagnosis of thyroid carcinoma was not excluded, and fine needle aspiration revealed non-diagnostic cytology. Of this reason, it was decided to follow up the nodule radiologically before second aspiration, which should have been performed after 3 months at the earliest. Two months later, the size of structure increased to $20 \mathrm{~mm} \times 21 \mathrm{~mm}$ in ultrasound examination. This time, surgical treatment was recommended because of the young age of the patient and notably increased size of the nodule in short period without repeated aspiration.

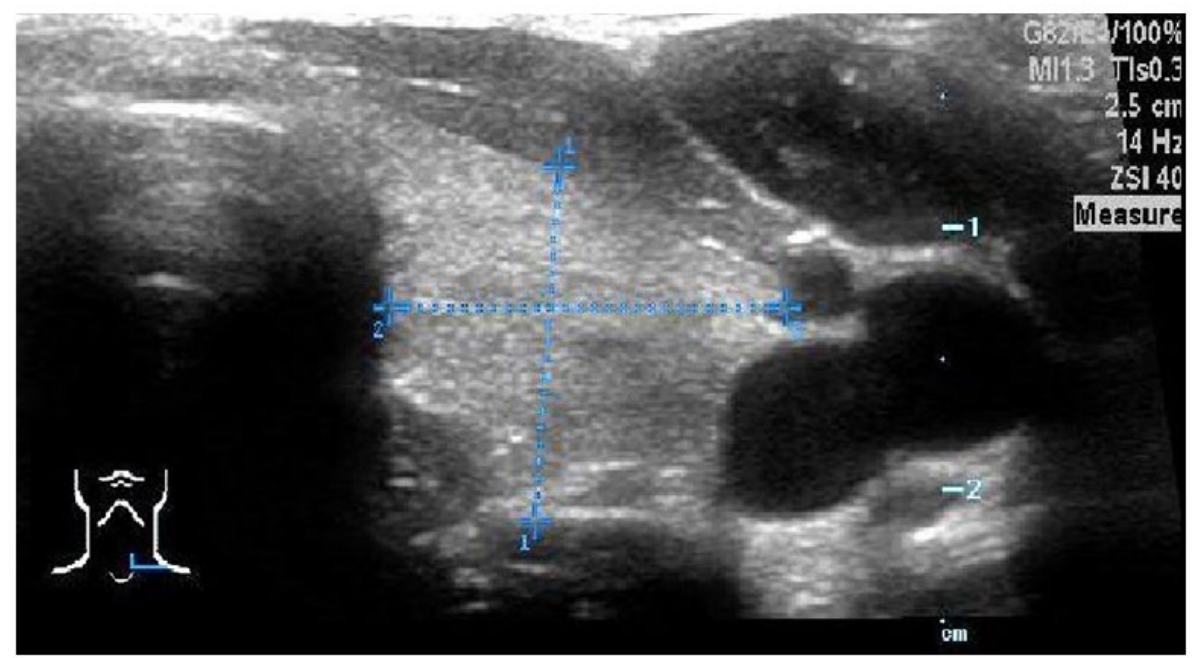

Figure 1. An ultrasound examination showing a hypoechogenic, well-vascularized structure, comparable to the thyroid tissue 


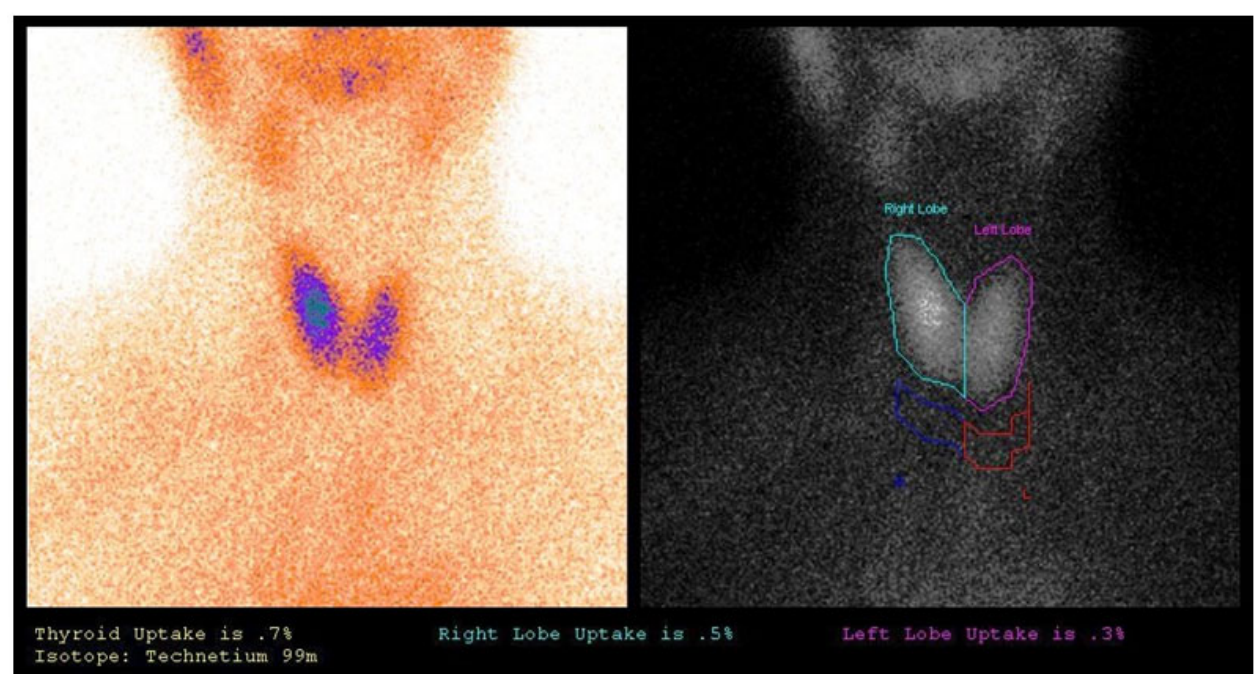

Figure 2. The SPECT test showing normal thyroid function

\subsection{Surgical treatment}

Informed patient consent had been obtained before the procedure. The patient was brought under general anesthesia with endotracheal intubation; surgery was performed by the cervicotomic approach. A resection of the pyramidal thyroid gland and isthmus was performed. Nodule with small amount of surrounding thyroid tissue was sent for urgent histological examination. The medullary thyroid carcinoma was suspected, and it was decided to perform thyroidectomy and bilateral central lymphadenectomy. The operation and early postoperative period showed no major complications. The patient was discharged from the hospital on the fourth postoperative day.

\subsection{Gross and histological features}

Fragment of thyroid gland measuring $4.0 \mathrm{~cm} \times 1.3 \mathrm{~cm} \times 1.3$ $\mathrm{cm}$ and weighing $6 \mathrm{~g}$ was sent for pathological examination. Grossly, the specimen contained brownish nodule $1.2 \mathrm{~cm}$ $\times 1.1 \mathrm{~cm} \times 1.2 \mathrm{~cm}$ in size, with small hemorrhages. The residual lobe $5 \mathrm{~cm} \times 2.5 \mathrm{~cm} \times 1 \mathrm{~cm}$, weighing $13 \mathrm{~g}$, and the other lobe $4.5 \mathrm{~cm} \times 2.5 \mathrm{~cm} \times 1 \mathrm{~cm}$, weighing $11 \mathrm{~g}$, did not show any macroscopic changes. Soft tissues of neck contained two lymph nodes, measuring $0.3 \mathrm{~cm}$ and $0.5 \mathrm{~cm}$.

Microscopically, encapsulated tumor had nested architecture composed of "zellballen" pattern with fibrovascular septation and moderate cellularity. Tumor was formed of medium sized epithelioid cells with slightly eosinophilic or clear cytoplasm and monomorphic, medium sized, round or oval, hyperchromic nuclei with low mitotic activity ( 1 mitosis / 50 HPF) (see Figure 3). Intravascular invasion in the capsule was observed. No necrosis was detected. Distance between tumor and thyroid follicles was $0.45 \mathrm{~mm}$. Surgical margins were free of tumor. Thyroid tissue and lymph nodes did not contain any significant changes.

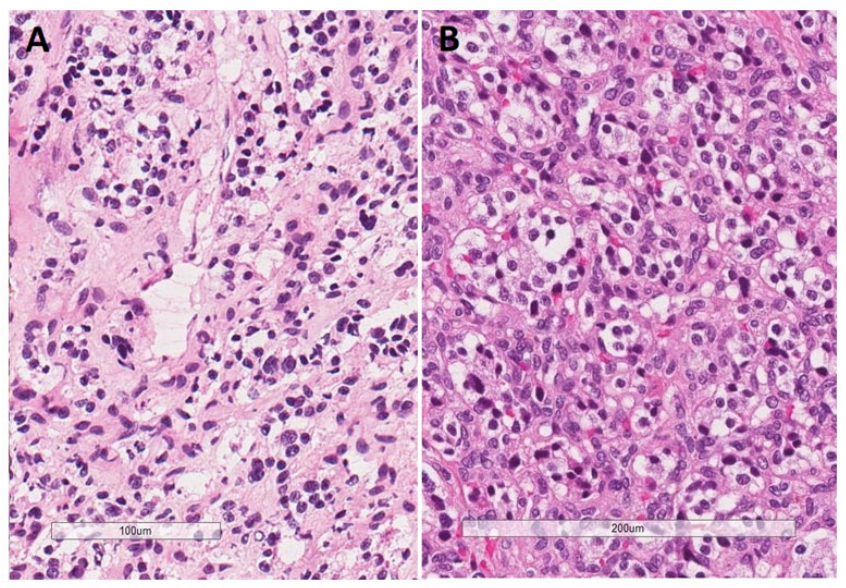

Figure 3. A. Intraoperative histology of paraganglioma (H\&E staining, $\times 200)$. B. Paraganglioma with nested architecture and distinct "zellballen" pattern separated by fibrovascular stroma and formed by epithelioid cells (H\&E staining, $\times 200)$.

Immunohistochemically, tumor cells showed strong positive granular cytoplasmic reaction for neuroendocrine markers (Synaptophysin and Chromogranin A) and were negative for Pancytokeratin (PanCK), TTF1, Thyroglobulin (THY), Thyroid peroxidase (TPO), PAX8, Calcitonin and polyclonal Carcinoembryonic Antigen (pCEA). Sustentacular cells, situated around tumor nests, were positive for S100. Ki67 proliferative activity was approximately $15 \%$ (see Figure 4). Staining for SDH mutations was performed as well. SDHA showed strong cytoplasmic reaction and SDHB showed moderate cytoplasmic reaction (see Figure 5), compared with internal and external (kidney tissue) controls. Therefore, germline mutations in SDH subunits were not suspected immunohistochemically. 


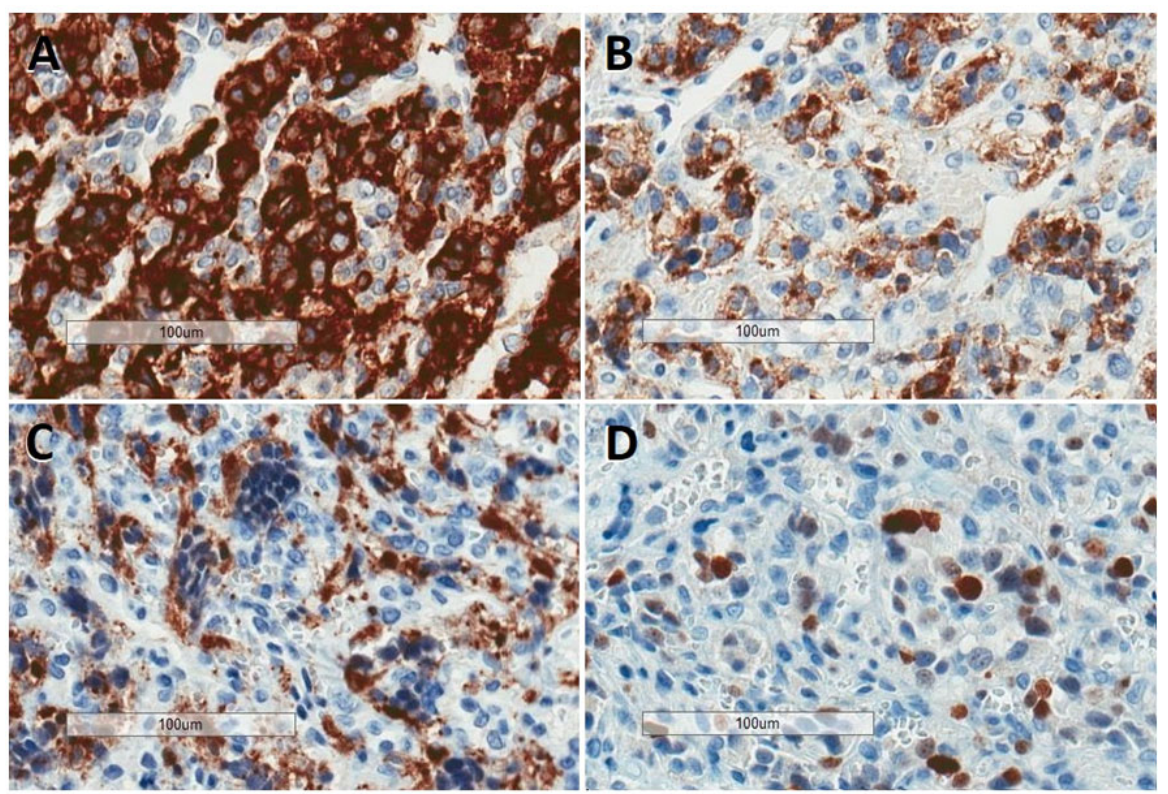

Figure 4. Immunohistochemical features in paraganglioma: A. Strong positive granular cytoplasmic reaction for Synaptophysin $(\times 200)$. B. Strong positive granular cytoplasmic reaction for Chromogranin $(\times 200)$. C. Positive reaction for S100 in sustentacular cells, situated around the tumor cells $(\times 200)$. D. Ki67 proliferative activity was approximately $15 \%$ $(\times 200)$.

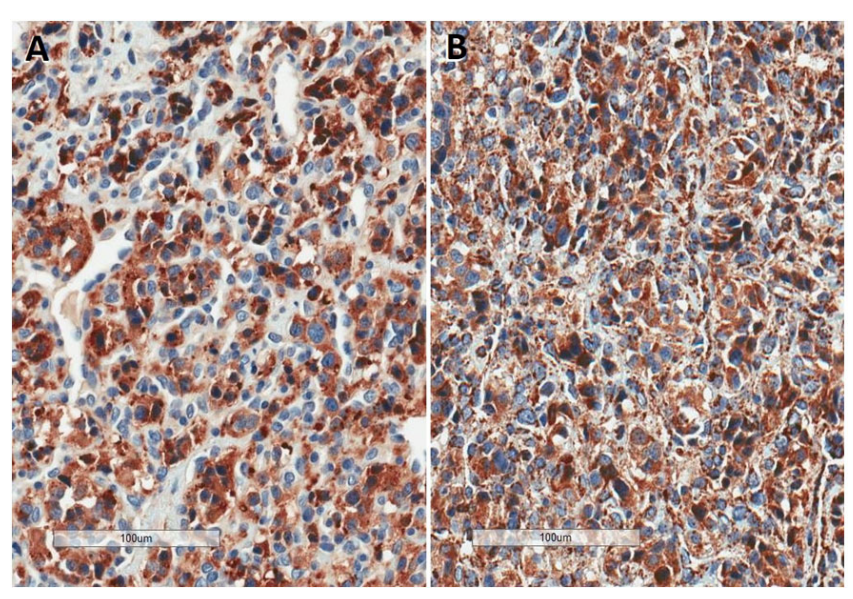

Figure 5. A. Strong positive granular cytoplasmic reaction for SDHA $(\times 200)$. B. Moderate positive granular cytoplasmic reaction for SDHB $(\times 200)$.

The conclusion of pathological examination was encapsulated paraganglioma $(12 \mathrm{~mm})$ in parathyroid soft tissue with intravascular invasion in the tumor capsule. Tumor was moderately differentiated, 4 points according Grading system for adrenal pheochromocytoma and paraganglioma (GAPP).

\subsection{Genetic evaluation}

Considering tumor presentation at early age, molecular genetic testing (NGS, Next Generation sequencing) was performed to identify associations with hereditary syndromes.
Test included VHL, RET, SDHB, SDHC, SDHD, SDHAF2, TMEM127, MAX, NF1, FH genes. Analysis revealed mutation in SDHB gene (c.213G $>$ A(p.Met71Ile) at 3 exon). Diagnosis of hereditary paraganglioma-pheochromocytoma syndrome (PGL4) was established.

\subsection{Further surveillance}

After PGL4 syndrome diagnosis, full patient examination for tumors in other localizations was performed. Periodic visits to endocrinologist were recommended. Surveillance of patient encompass clinical and biochemical evaluation for abdominal, head and neck PGL, RCC and GIST: 1) urinary metanephrines and catecholamines testing and/or plasma metanephrines testing annually; 2) neck-chest-abdominalpelvic CT, MRI, 123I-MIBG +/- octreotide scintigraphy or PET scan, if catecholamines are elevated; 3) neck-chestabdominal-pelvic CT or MRI scan every two years; 4) 123IMIBG scintigraphy every four or five years.

Currently, more than nine months after the surgery, the patient is asymptomatic, without any laboratory or radiologic evidence. In the near future, the neck-chest-abdominal-pelvic PET scan is going to be performed one year after surgery to exclude and monitor any residual, recurrent or newly arising tumor.

\section{Discussion}

Head and neck paragangliomas are rare tumors, which covers only $0.012 \%$ of all head and neck tumors and less than $3 \%$ 
of all paragangliomas. ${ }^{[1]}$ The diagnosis of paraganglioma is difficult to confirm by using ultrasound sonography or computed tomography (CT) scan. Fine needle aspiration mostly helps to exclude malignant tumors of thyroid but does not approve diagnosis of paraganglioma. Radiologically, PG typically shares similar features as common types of thyroid neoplasms. Tumor appears as a solid hypoechogenic nodule on ultrasound sonography and as a low-density mass on CT scans. ${ }^{[2,15]}$

In our case, PG was closely located to thyroid gland. Microscopically, distance between thyroid tissue and the tumor was only $0.45 \mathrm{~mm}$. Therefore, its evaluation was difficult in intraoperative frozen section analysis. Of these reasons, the nodule has been considered as thyroid tumor at first.

Regarding to anatomical localization of our represented tumor, the differential diagnosis was focused in primary thyroid tumors. Because of PGs rarity in this localization, intraoperative frozen section analysis rarely results in correct diagnosis. ${ }^{[2,5,16]}$ Cases from thyroid gland mostly indicate various diagnoses of thyroid tumors, but PGs in this localization are rarely correctly diagnosed at first..$^{[2,17]}$ Kim et al. ${ }^{[5]}$ posted in retrospective study that none of the 7 PGs cases in the thyroid gland were diagnose correctly during intraoperative frozen section analysis. These cases were misdiagnosed with medullary thyroid carcinoma $(57 \%, 4 / 7)$, thyroid carcinoma $(14.3 \%, 1 / 7)$, follicular carcinoma $(14.3 \%, 1 / 7)$ and Hürthle cell carcinoma $(14.3 \%, 1 / 7)$.

In our case, medullary thyroid carcinoma was the most challenging part, due to architectural similarities. Medullary carcinoma may mimic paraganglioma (paraganglioma likevariant) with nested architecture, low cytological atypia and expression of neuroendocrinal differentiation. ${ }^{[18]}$ These two entities are separated only by immunohistochemical staining: medullary thyroid carcinoma is mostly positive for epithelial markers, Calcitonin, CEA, TTF1 and PAX8. The lack of S100 positive sustentacular cells in medullary carcinoma also facilitate differential diagnosis. A distinction between these neoplasms is essential, because management and follow-up strategy vary greatly. ${ }^{[2,6]}$

Further differential diagnosis includes hyalinizing trabecular tumor, also known as paraganglioma like adenoma. This peculiar type of follicular cell neoplasm is formed by characteristic trabecular structures with prominent intratrabecular hyalinization and delicate vascularization. By immunohistochemistry, tumor is positive for Thyroglobulin, TTF1 and shows unique expression of Ki-67 in cell membranes. Another difficulty is parathyroid adenoma of its histological appearance and neuroendocrinal differentiation. Fortunately, only parathyroid adenoma is positive for Parathyroid Hor- mone (PTH) immunostaining. ${ }^{[16,19]}$

In addition, atypical follicular carcinoma or oncocytic (Hürthle cell) carcinoma are less confusing. In contrast to paraganglioma, these neoplasms of thyroid gland will be positive for epithelial markers, Thyroglobulin, TTF1 and PAX8. Moreover, the differential diagnosis for neck paragangliomas includes lymph nodes that may harbor metastatic tumor especially other neuroendocrine tumors like Merkle cell carcinoma. All these entities will be positive for Cytokeratins and residual lymphoid tissue may be observed. ${ }^{[1,2]}$

In this case, a system called grading system for adrenal pheochromocytoma and paraganglioma (GAPP) ${ }^{[20]}$ was used to predict the risk of metastasis. GAPP criteria include histological pattern, cellularity, comedo type-necrosis, capsular or vascular invasion, Ki67 labelling index and catecholamine production. Our tumor was scored with 4 points (1 point for moderate cellularity, 1 point for presence of vascular or capsular invasion and 2 points for Ki67 labelling index higher than 3\%) and assigned to moderately differentiated type. Although, GAPP is not reliable enough and it has been noticed that inclusion of SDHB staining to GAPP may help to increase accuracy to predict risk of metastasis. ${ }^{[21]}$

Head and neck paragangliomas are associated with SDH mutations in $40 \%$ of cases. $^{[1,22]}$ The utility of SDHA immunohistochemistry may be limited in this cohort. ${ }^{[23]}$ Generally, SDH mutations in the head and neck paragangliomas occur in SDHB, SDHD, SDHC and rarely SDHAF2 genes. When one or more of these genes are mutated, SDHB immunohistochemical staining is negative. ${ }^{[3,8,24]}$ SDHB immunohistochemistry is considered as reliable method of its high sensitivity, which can reach almost 95\%, and sufficient specificity, which is higher than $80 \%$. Although, not all SDH mutated tumors like in our represented case will show negative staining. Therefore, genetical consultation should be recommended considering young age and other significant indications. ${ }^{[3,13,14,25]}$

Although, paragangliomas are still considered as tumors with uncertain malignant potential, the specific genetic alterations allow distinction of risk of aggressive disease. SDHB mutated tumors has highest risk of metastasis and, therefore, overall survival is poor with $11 \%-36 \%$ in 5 years, though it is more important in chest-abdominal-pelvic localizations. ${ }^{[1,26,27]}$ Head and neck paragangliomas have higher risk for SDHD mutation carriers (60\%-79\%), but SDHB mutated head and neck paragangliomas may also have potential for malignancy. ${ }^{[11]}$ Although, previously SDHB negative tumors in this localization were considered as completely benign ${ }^{[10]}$ they may metastasize, be multiple (15\%) or even have associations with pheochromocytomas $(2 \%)$ and other 
non-paraganglionic tumors $(3 \%)$ in some cases. ${ }^{[11,12]}$ Of these reasons, SDHB mutated head and neck tumors do not have to be single out entirely from SDHB mutated tumors in other localizations, considering further surveillance. ${ }^{[11]}$

After detection of genetical mutations in SDH family genes, patients with hereditary PG syndromes should be closely observed not only for potential tumor recurrence, multifocality and distant metastases, but also newly arising tumors in other typical locations. ${ }^{[3,8,27,28]}$ Active multicomplex surveillance may help to diagnose neoplasm in early stages, manage them with surgical treatment and prevent spreading of malignancies. $^{[29]}$
In conclusion, the diagnosis of neck paragangliomas, which are close to thyroid gland, may be difficult based on preoperative radiological imaging and histological evaluation without immunohistochemical staining.

Secondary, the possibility of false positive SDHB staining in paragangliomas should not be ruled out and genetic testing must be recommended according other indications like young age, because hereditary paragangliomas syndromes requires careful monitoring of the patient.

\section{CONFLICTS OF InTEREST Disclosure}

The authors declare that they have no competing interest.

\section{REFERENCES}

[1] Williams MD. Paragangliomas of the Head and Neck: An Overview from Diagnosis to Genetics. Head Neck Pathol. 2017 Sep; 11(3) 278-87. PMid:28321772. https://doi.org/10.1007/s12105 $-017-0803-4$

[2] Yu X, Wang Y, Wang P, et al. Primary thyroid paraganglioma mimicking medullary thyroid carcinoma: A case report. Oncol Lett. 2015 Aug; 10(2): 1000-2. PMid:26622613. https://doi.org/10.389 2/ol.2015.3292

[3] Gill AJ. Succinate dehydrogenase (SDH)-deficient neoplasia Histopathology. 2018 Jan; 72(1): 106-16. PMid:29239034. https : //doi.org/10.1111/his.13277

[4] Aydoğan Bİ, Dizbay Sak S, Güllü S. Cervical Paraganglioma Mimicking Thyroid Nodule: A Rare Clinical Case. Case Rep Endocrinol [Internet]. 2016; 2016. Available from: https://www.ncbi.nlm .nih.gov/pmc/articles/PMC4811066/

[5] Kim B, Yoo Y, Lee J, et al. Pitfalls of Frozen Section Diagnosis for Paraganglioma: A Clinicopathologic Analysis and Review of the Literature. Int J Surg Pathol. 2018 May; 26(3): 213-20.

[6] Lloyd RV, Osamura RY, Kloppel G, et al. World Health Organization classification of tumours. Pathology and genetics of tumours of endocrine organs. Lyon: IARS Press; 2017.

[7] Fishbein L. Pheochromocytoma and Paraganglioma: Genetics, Diagnosis, and Treatment. Hematol Oncol Clin North Am. 2016 Feb; 30(1): 135-50. PMid:26614373.

[8] Gill AJ. Succinate dehydrogenase (SDH) and mitochondrial driven neoplasia. Pathology. 2012 Jun; 44(4): 285-92. PMid:22544211. https://doi .org/10.1097/PAT.0b013e3283539932

[9] Barletta JA, Hornick JL. Succinate dehydrogenase-deficient tumors: diagnostic advances and clinical implications. Adv Anat Pathol. 2012 Jul; 19(4): 193-203. PMid:22692282. https : //doi .org/10.109 7/PAP.0b013e31825c6bc6

[10] Persu A, Hamoir M, Grégoire V, et al. High prevalence of SDHB mutations in head and neck paraganglioma in Belgium. J Hypertens. 2008 Jul; 26(7): 1395-401.

[11] Rijken JA, Niemeijer ND, Leemans CR, et al. Nationwide study of patients with head and neck paragangliomas carrying SDHB germline mutations. BJS Open. 2018 Apr; 2(2): 62-9.

[12] Ohtake M, Tateishi K, Murata H, et al. Succinate Dehydrogenase B Subunit-Negative Jugular Foramen Paraganglioma Manifesting Malignant Progression with Pseudohypoxia-Related Atypical Uptake of [18F]-Fluoro-2-Deoxy-d-Glucose: A Case Report. World Neurosurg.
2018 Jun; 114: 47-52. PMid:29510284. https ://doi .org/10.1 016/j.wneu . 2018.02.147

[13] Andrici J, Gill AJ, Hornick JL. Next generation immunohistochemistry: Emerging substitutes to genetic testing? Semin Diagn Pathol. 2018 May; 35(3): 161-9. PMid:28662997. https ://doi.org/10 $.1053 / \mathrm{j}$. semdp. 2017.05.004

[14] Udager AM, Magers MJ, Goerke DM, et al. The utility of SDHB and FH immunohistochemistry in patients evaluated for hereditary paraganglioma-pheochromocytoma syndromes. Hum Pathol. 2018 Jan; 71: 47-54.

[15] Schmit GD, Gorman B, van Heerden JA, et al. Inferior laryngeal paraganglioma mimicking a primary thyroid tumor. Endocr Pract. 2006 Aug; 12(4): 432-5. PMid:16901801. https ://doi.org/10 $.4158 / \mathrm{EP} .12 .4 .432$

[16] Yu BH, Sheng WQ, Wang J. Primary paraganglioma of thyroid gland: a clinicopathologic and immunohistochemical analysis of three cases with a review of the literature. Head Neck Pathol. 2013 Dec; 7(4): 373-80. PMid:23943066. https://doi.org/10.100 7/s12105-013-0467-7

[17] Nguyen NA, Peter I, Levay B, et al. Thyroid gland paraganglioma: report of a case and review of the literature. Int J Clin Exp Med. 2017; 10(12): 16703-16708.

[18] Bockhorn M, Sheu SY, Frilling A, et al. Paraganglioma-like medullary thyroid carcinoma: a rare entity. Thyroid. 2005 Dec; 15(12): 1363-7. PMid:16405409. https://doi.org/10.1089/ thy.2005.15.1363

[19] Yano Y, Nagahama M, Sugino K, et al. Paraganglioma of the thyroid: report of a male case with ultrasonographic imagings, cytologic, histologic, and immunohistochemical features. Thyroid. 2007 Jun; 17(6): 575-8. PMid:17614779. https://doi.org/10.1089/thy . 2006.0284

[20] Kimura N, Takayanagi R, Takizawa N, et al. Pathological grading for predicting metastasis in phaeochromocytoma and paraganglioma. Endocr Relat Cancer. 2014 Jun; 21(3): 405-14.

[21] Nicolas M, Dahia P. Predictors of outcome in phaeochromocytomas and paragangliomas. F1000Res [Internet]. 2017 Dec 21; 6. Available from: https://www.ncbi.nlm.nih.gov/pmc/articles/PMC5 749134/

[22] Dahia PLM. Pheochromocytoma and paraganglioma pathogenesis: learning from genetic heterogeneity. Nat Rev Cancer. $2014 \mathrm{Feb}$; 14(2): 108-19. PMid:24442145. https://doi.org/10.1038/nr c3648 
[23] Korpershoek E, Favier J, Gaal J, et al. SDHA immunohistochemistry detects germline SDHA gene mutations in apparently sporadic paragangliomas and pheochromocytomas. J Clin Endocrinol Metab. 2011 Sep; 96(9): E1472-1476.

[24] Gill AJ, Benn DE, Chou A, et al. Immunohistochemistry for SDHB triages genetic testing of SDHB, SDHC, and SDHD in paraganglioma-pheochromocytoma syndromes. Hum Pathol. 2010 Jun; 41(6): 805-14.

[25] Santi R, Rapizzi E, Canu L, et al. Potential Pitfalls of SDH Immunohistochemical Detection in Paragangliomas and Phaeochromocytomas Harbouring Germline SDHx Gene Mutation. Anticancer Res. 2017; 37(2): 805-12. PMid:28179334. https ://doi .org/10 .21873/anticanres.11381
[26] Lee JH, Barich F, Karnell LH, et al. National Cancer Data Base report on malignant paragangliomas of the head and neck. Cancer. 2002 Feb 1; 94(3): 730-7.

[27] Sethi RV, Sethi RKV, Herr MW, et al. Malignant head and neck paragangliomas: treatment efficacy and prognostic indicators. Am J Otolaryngol. 2013 Oct; 34(5): 431-8. PMid:23642313. https: //doi.org/10.1016/j.amjoto.2013.03.010

[28] Ellis RJ, Patel D, Prodanov T, et al. The presence of SDHB mutations should modify surgical indications for carotid body paragangliomas. Ann Surg. 2014 Jul; 260(1): 158-62. PMid:24169168. https://doi.org/10.1097/SLA. 0000000000000283

[29] Lenders JWM, Duh QY, Eisenhofer G, et al. Pheochromocytoma and paraganglioma: an endocrine society clinical practice guideline. J Clin Endocrinol Metab. 2014 Jun; 99(6): 1915-42. 